\title{
Estrés oxidativo y alteraciones de la funcionalidad hepática en ratones hembras con hígado graso experimental
}

\author{
López-Ortega, A.A.'; Aranguren, A.J.'; Plaza, M.A.'; Murillo, M.D. ${ }^{2}$
}

'Unidad de Investigación en Ciencias Funcionales "Dr. H. Moussatché" (UNIHM), Facultad de Ciencias Veterinarias, UCLA, Ap. Postal 267, Barquisimeto 3001, Venezuela, Teléfono (251) 2592409, Fax 2592404,

E-mail: alopez@ucla.edu.ve. Departamento Farmacología y Fisiología, Facultad de Veterinaria,

Universidad de Zaragoza, España.

\begin{abstract}
Resumen
López-Ortega, A.A.; Aranguren, A.J.; Plaza, M.A.; Murillo, M.D.: Estrés oxidativo y alteraciones de la funcionalidad hepática en ratones hembras con hígado graso experimental. Rev. vet. 25: 1, 7-11, 2014. El estrés oxidativo (EO) afecta la progresión y severidad de diferentes patologías, entre las cuales se incluyen las hepáticas. Ha sido señalada su intervención en el hígado graso (HG) no alcohólico, de relevante importancia tanto en medicina humana como veterinaria debido, entre otras, a la alteración de las funciones hepáticas causada por el deterioro celular producto del EO, como es el proceso de lipoperoxidación. El objetivo de este estudio fue determinar si el HG no alcohólico experimental inducido en ratones hembra NMRI por etionina provocaba EO y afectaba a la función hepática. El HG se indujo mediante la administración de etionina $(7,5 \mathrm{mg} / 20 \mathrm{~g}$ peso corporal), de conocida acción hepatotóxica. Se utilizaron dos grupos de 10 animales: uno control y otro tratado con DL-etionina. El HG se evaluó por métodos histológicos y mediante la cuantificación de los triglicéridos hepáticos, que indicaron hepatoesteatosis en las hembras inyectadas con etionina. La concentración hepática de malondialdehído (MDA) y de dienos conjugados (DC) se determinó por espectrofotometría como parámetros de EO. Además, en muestras de plasma se cuantificó la concentración de las aminotransferasas ALT y AST a través de kits comerciales. La inducción de HG causó una elevación significativa de los parámetros de MDA: de $258,30 \pm 22,49 \mathrm{nmoles} / \mathrm{mg}$ proteínas a $354,90 \pm 19,83 \mathrm{nmoles} / \mathrm{mg}$ proteínas $(\mathrm{p}<0,01)$, así como los de DC: de $212,46 \pm 8,36 \mu$ moles/mg proteínas a $263,93 \pm 14,51 \mu$ moles $/ \mathrm{mg}$ proteínas $(\mathrm{p}<0,01)$. En el HG, la actividad plasmática de las aminotransferasas aumentó significativamente: ALT de 77,01 $\pm 4,11 \mathrm{U} / 1$ a $198,34 \pm 17,22 \mathrm{U} / 1(\mathrm{p}<0,001)$ y AST de $188,29 \pm 11,81 \mathrm{U} / 1$ a $401,71 \pm 22,42 \mathrm{U} / 1(\mathrm{p}<0,001)$. Se concluye que el HG inducido por etionina se acompaña con un estado de EO que compromete la funcionalidad hepática.
\end{abstract}

Palabras clave: ratón, hígado graso, etionina, estrés oxidativo, disfunción.

\begin{abstract}
López-Ortega, A.A.; Aranguren, A.J.; Plaza, M.A.; Murillo, M.D.: Oxidative stress and alterations in liver function in female mice with experimental fatty liver. Rev. vet. 25: 1, 7-11, 2014. The oxidative stress (OS) affects the progression and severity of different pathologies, including those affecting the liver). OS has been associated with the non-alcoholic fatty liver (NAFL), of importance in both human and veterinary medicine due to hepatic disfunction as a consequence of cellular deterioration, part of the lipid peroxidation process. The objective of this study was to determine if experimental NAFL in female NMRI mice induced by ethionine evokes OS and alters hepatic function. Fatty liver (FL) was induced by the administration of ethionine $(7.5 \mathrm{mg} / 20 \mathrm{~g}$ body weight), of known hepatotoxic effects. Two groups of 10 animals each, were used (control and injected with DL-ethionine). FL was assessed by histology and the quantification of hepatic triglycerides, which indicated hepatic steatosis. For the determination of OS, malondialdehyde (MDA) and conjugated diene (CD) concentrations were determined by means of spectrophotometry. In addition, the concentrations of ALT and AST in plasma were quantified using commercial kits. The induction of FL caused significant elevation of liver parameters indicative of OS, MDA: from $258.30 \pm 22.49$ nmoles $/ \mathrm{mg}$ protein to $354.90 \pm 19.83 \mathrm{nmoles} / \mathrm{mg}$ protein $(\mathrm{p}<0.01)$, as well as DC: from 212.46 $\pm 8.36 \mu \mathrm{moles} / \mathrm{mg}$ protein to $263.93 \pm 14.51 \mu \mathrm{moles} / \mathrm{mg}$ protein $(\mathrm{p}<0.01)$. In FL the plasmatic activity of aminotransferases significantly increased: ALT from $77.01 \pm 4.11 \mathrm{U} / 1$ to 198.34 $\pm 17.22 \mathrm{U} / 1(\mathrm{p}<0.001)$ and AST from $188.29 \pm 11.81 \mathrm{U} / 1$ to $401.71 \pm 22.42 \mathrm{U} / 1(\mathrm{p}<0.001)$. It is
\end{abstract}

Recibido: 26 febrero 2014 / Aceptado: 3 abril 2014 
concluded that the ethionine-induced FL is accompanied with a state of OS which compromises the liver function.

Key words: mouse, fatty liver, ethionine, oxidative stress, dysfunction.

\section{INTRODUCCIÓN}

El depósito de grasa en el hígado de origen no alcohólico (HGNA) corresponde a una patología inflamatoria que se inicia con hepatoesteatosis, estado benigno de carácter reversible, el cual, al persistir la causa inductora evoluciona a una esteatohepatitis crónica que puede llevar a la etapa final fibrótica de esta enfermedad. El HGNA es relevante en medicina humana debido a su relación con la obesidad y la diabetes ${ }^{16}$, como también en medicina veterinaria en la cual el hígado graso (HG) afecta a diferentes especies animales, entre ellas al ganado bovino productor de leche, particularmente en el período del periparto, lo que causa pérdidas importantes en el sector agropecuario ${ }^{2}$.

El estrés oxidativo (EO) está involucrado en los procesos inflamatorios, es así como en las enfermedades del hígado se reportan altas concentraciones de especies reactivas del $\mathrm{O}_{2}$ (ROS) ${ }^{11}$. El objetivo de este estudio fue determinar si el HG no alcohólico experimental inducido en ratones hembra NMRI por DLetionina provocaba EO y afectaba a la función hepática.

\section{MATERIAL Y MÉTODOS}

Población y muestra. Para el estudio se utilizaron ratones hembras provenientes de la población de ratones de la cepa NMRI del Bioterio Central de la UCLA, Venezuela. Mediante muestreo aleatorio simple, se seleccionaron 20 ratones adultos de 27,5 $\pm 2,5 \mathrm{~g}$ de peso corporal. Colocados en jaulas individuales de acero inoxidable, bajo ciclos de 12 horas/12 horas luz/oscuridad en las condiciones estandarizadas del Bioterio de la UNIHM, con libre acceso al agua y a un alimento comercial para ratones Ratarina ${ }^{\circledR}$ (Protinal, Venezuela). El estudio fue conducido de acuerdo al Código de Bioética y Bioseguridad de FONACIT-Venezuela, 2009.

Manejo de animales y toma de muestras. Los ratones fueron distribuidos aleatoriamente en dos gru- pos de 10 animales cada uno: un grupo control y otro inyectado con DL-etionina (Sigma Chemical Co, St Louis, MO, EE.UU.) disuelta en solución fisiológica estéril, administrada i.p. en dosis de 7,5 mg/20 g de peso corporal. Los dos grupos fueron sometidos a igual manejo excepto que a los controles se les inyectó sólo el vehículo (solución fisiológica estéril). Luego de un ayuno de 48 horas, por venopunción coccígea se obtuvo una muestra de sangre tratada con anticoagulante EDTA-Na al $2 \%$. Los plasmas se separaron por centrifugación ( $800 \mathrm{~g}, 20$ minutos) en microcentrífuga refrigerada Eppendorf 5402 (Brinkman Instr., Westbury, NY, EE.UU.) y fueron mantenidos a $4^{\circ} \mathrm{C}$.

Obtenida la muestra de sangre, a los animales se les practicó la eutanasia bajo ligera anestesia con éter. Se disecó el hígado, se evaluó su aspecto macroscópico y se sumergió en tampón Tris-sacarosa $250 \mathrm{mM} \mathrm{pH} \mathrm{7,2} \mathrm{a}$ $4^{\circ} \mathrm{C}$. Se homogeneizaron $1,5 \mathrm{~g}$ de hígado con 3 veces su peso en buffer Tris-sacarosa a $4^{\circ} \mathrm{C}$ en un potter NSI-12 Bodine (Electric Comp., Chicago, ILL, EE.UU.). El sobrenadante del homogeneizado, diluido 1:40 con agua desionizada, se mantuvo a $4^{\circ} \mathrm{C}$ hasta su procesamiento.

Estudio histopatológico. Una pequeña porción de hígado fijado en formol tamponado al $10 \%$, fue utilizada para su estudio histopatológico realizado en el Laboratorio de Anatomía Patológica del Hospital Veterinario "Dr. Humberto Ramírez Daza" de la Facultad de Ciencias Veterinarias-UCLA. Se obtuvieron secciones de 4 micrómetros del tejido mediante el uso de un micrótomo Jung AG (Heidelberg, Alemania) y posterior tinción de los cortes con hematoxilina-eosina de acuerdo a la técnica convencional.

Valoración de los triglicéridos y del estrés oxidativo. En el sobrenadante diluido del homogeneizado hepático se determinó la concentración de triglicéridos por método enzimático mediante kit de Qualitest (Ind. Qualitest, Caracas, Venezuela). Los resultados se expresaron en $\mathrm{mg}$ de triglicéridos/mg de proteínas,
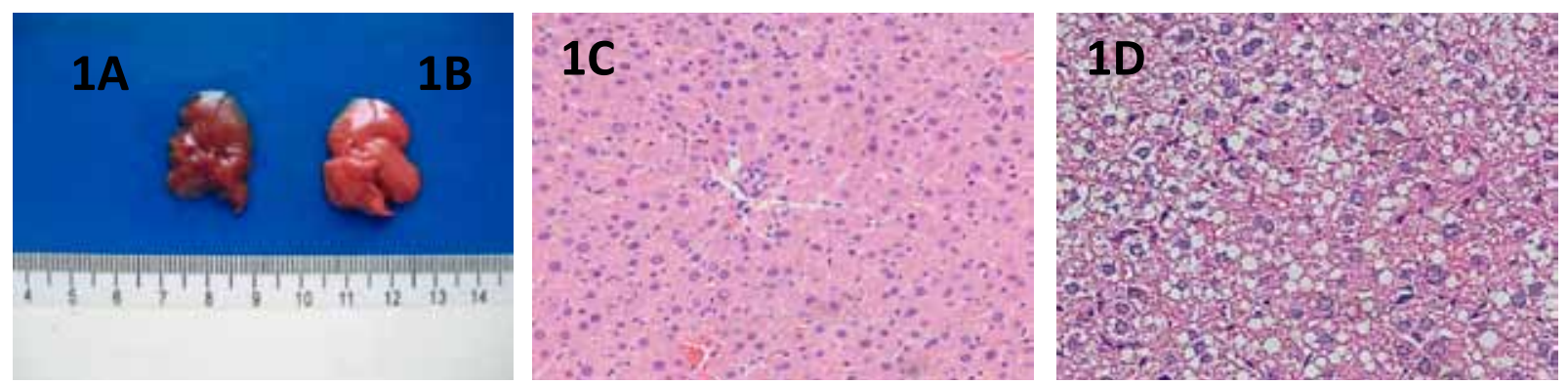

Figura 1. 1A y 1B: hígados de ratones controles y con HG inducido por DL-etionina, respectivamente. 1C y 1D: fotomicrografías de hígados control e HG inducido, respectivamente. H\&E 40X. 
las cuales fueron determinadas mediante el kit Pierce (Rockford, ILL, EE.UU.) basado en la técnica descrita por Bradford ${ }^{4}$. Igualmente en este sobrenadante, se determinó la concentración de dienos conjugados (DC) a través de la técnica descrita por Wallin y col ${ }^{14} \mathrm{y}$ también la concentración de malondialdehído (MDA) mediante el método de TBARS (sustancias reaccionantes con el ácido 2-tio barbitúrico) de acuerdo a Ohkawa y $\operatorname{col}^{10}$.

Valoración de la funcionalidad hepática. En el plasma fueron determinadas las actividades de alanina amino-transferasa (ALT) y aspartato aminotransferasa (AST), mediante kits comerciales (Wiener Lab, Rosario, Argentina). Todas las lecturas espectrofotométricas se llevaron a cabo en un Genesys 5 (Rochester, NY, EE.UU.).

Análisis estadístico. Se realizó mediante el programa SPSS versión 17.0 para Windows. Los resultados quedaron expresados como el promedio de los valores \pm error estándar (ES). Para establecer la significación estadística de los resultados se utilizó la prueba " $\mathrm{t}$ " de Student exigiéndose un 95\% de certeza $(\mathrm{p}<0,05)$. ratones inyectados con DL-etionina con respecto a los controles, tal como indica la Figura 3.

Funcionalidad hepática. Las actividades plasmáticas de alanina amino-transferasa (ALT) y aspartato aminotransferasa (AST), aumentaron significativamente $(p<0,001)$ en ratones con HG inducido por DLetionina (Figura 4).

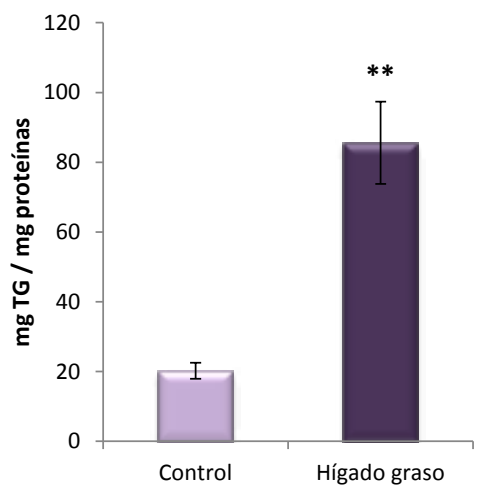

Figura 2. Concentración de triglicéridos en hígado de ratones controles y con $\mathrm{HG}$ inducido (media $\pm \mathrm{ES}$ de 10 animales/grupo). ${ }^{* *} \mathrm{p}<0,01$ con respecto al grupo control.

\section{RESULTADOS}

Hepatoesteatosis. En la Figura 1 se observa el aspecto macroscópico del hígado de ratón hembra NMRI adulto inyectado con DLetionina, el cual presenta color amarillo (B) a diferencia de la tonalidad roja del animal control (A).

En los estudios histopatológicos presentados en la Figura 1, los animales inyectados con DL-etionina revelan una metamorfosis grasa severa, caracterizada por hepatocitos con numerosas vacuolas lipídicas citoplasmáticas (D) en contraposición con el aspecto normal del hígado control (C).

La concentración de triglicéridos hepáticos / mg proteínas, se mostró aumentada significativamente $(p<0,01)$ en los ratones con hígado graso inducido por DL-etionina, en relación al valor control (Figura 2), lo cual corrobora los hallazgos histopatológicos obtenidos en este estudio (Figura 1D).

Estrés oxidativo hepático. En el presente estudio, la concentración hepática de los parámetros para valorar el EO (dienos conjugados y malondialdehído) aumentó significativamente $(\mathrm{p}<0,01)$ en los
A

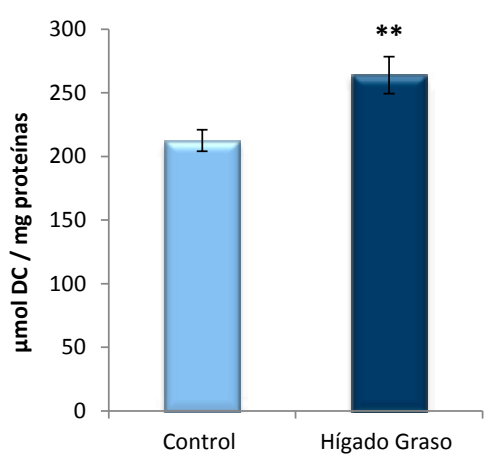

Figura 3. Concentración hepática de dienos conjugados (A) y malondialdehído (B) en ratones controles y con hígado graso inducido por DLetionina (media \pm ES de 10 animales/grupo). ** $\mathrm{p}<0,01$ con respecto al grupo control.

A

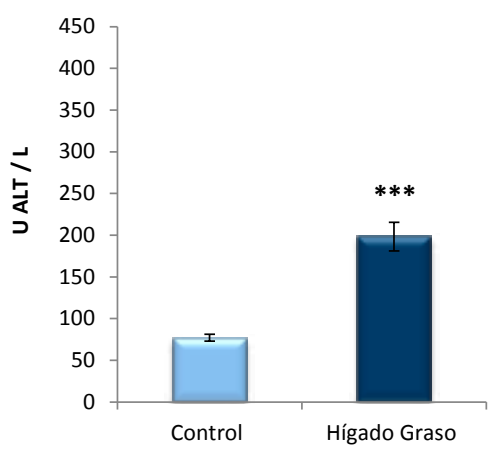

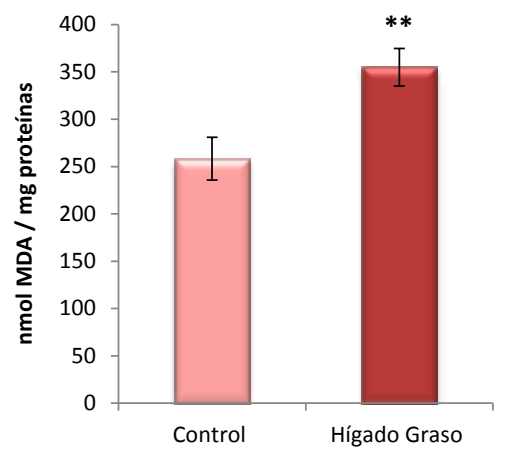

Figura 4. Actividad plasmática (U/L) de las aminotransferasas ALT (A) y AST (B) en ratones controles y con HG inducido por DL-etionina (media \pm ES de 10 animales/grupo). $* * * \mathrm{p}<0,001$ con respecto al grupo control. 


\section{DISCUSIÓN}

El HG es una patología relevante en medicina veterinaria, ya que compromete la salud y producción animal, con un impacto económico negativo en el sector agropecuario. En bovinos lecheros es considerada la mayor alteración metabólica del periparto, con disminución de la ingesta de alimento y de la producción de leche, a lo que se añade el costo del tratamiento del $\mathrm{HG}^{2}$. Para cubrir las demandas energéticas, el animal debe movilizar las reservas lipídicas, particularmente desde el tejido adiposo. El exceso de lípidos movilizados se acumula en las células del hígado en forma de triglicéridos ${ }^{15}$.

De igual manera, el HGNA es relevante en medicina humana debido a su relación con la obesidad y la diabetes ${ }^{16}$. Recientemente se ha determinado que una dieta rica en lípidos induce depósito graso en los tejidos hepático y esquelético con simultánea resistencia a la insulina; el estrés oxidativo y la disfunción mitocondrial están involucrados en el proceso ${ }^{17}$.

Al progresar el HGNA puede evolucionar hacia una esteatohepatitis y finalmente concluir en una cirrosis hepática o un carcinoma hepatocelular, como se ha establecido experimentalmente en modelos animales (ratones y ratas) con la utilización de diferentes inductores ${ }^{13}$.

La etionina es un etil-análogo del aminoácido metionina y compite con ésta en las diferentes rutas metabólicas en que interviene. La transformación ocurre en el hígado de la mayoría de los mamíferos, por acción de la metionina-adenosiltransferasa que cataliza su conversión a S-adenosil-metionina ${ }^{9}$. La falta de esta enzima causa una drástica disminución del ATP hepático con la subsecuente disminución de la síntesis proteica ${ }^{12}$. Entre otras se verá disminuida la formación de las lipoproteínas de muy baja densidad (VLDL), macromoléculas encargadas de la exportación de lípidos desde el hígado a la sangre, lo que origina el acúmulo graso en el hepatocito.

En el presente estudio los ratones inyectados con DL-etionina presentaron un hígado con metamorfosis grasa severa: los hepatocitos mostraron un citoplasma con numerosas vacuolas lipídicas y formación de microquistes lipídicos producto de la ruptura de la membrana plasmática con fusión de las vacuolas (Figura 1D).

Aunque diferentes tipos de lípidos pueden acumularse en el hígado debido a la acción de hepatotóxicos, los triglicéridos (TG) son los principalmente involucrados en este depósito ${ }^{7}$, hecho coincidente con los resultados del presente estudio, tal como se muestra en la Figura 2, en la cual se observa que la concentración de TG está muy aumentada en el homogeneizado del hígado de animales inyectados con DL-etionina, en comparación con el nivel mostrado por los controles. Los cuerpos lipídicos no son almacenajes estáticos de grasa en el hepatocito sino que poseen un metabolismo muy activo y una composición similar a la de una lipoproteína. Presentan un núcleo formado por lípidos hidrofóbicos (TG y en menor proporción colesterol esterificado) asociados a una monocapa de lípidos polares (fosfolípidos y colesterol libre) y proteínas ${ }^{5}$.

La generación de radicales libres (RL) es necesaria para diferentes funciones celulares. La elevación de la concentración de RL define un estado de estrés oxidativo que induce alteraciones del metabolismo celular, incluyendo modificaciones de la estructura de proteínas y ácidos nucleicos, así como un incremento del ión calcio intracelular. Además, altera el transporte iónico de la membrana y su permeabilidad, produciendo destrucción celular por el proceso de lipoperoxidación, mecanismo que altera la estructura normal de los lípidos de membrana ${ }^{8}$.

Entre las biomoléculas dañadas por los RL se encuentran los ácidos grasos poli-insaturados, los cuales sufren una secuencia lipoperoxidativa (LPO), entre cuyos productos iniciales están los DC y entre los finales se encuentra, junto a otros aldehídos, el MDA. Estos productos de la degradación lipoperoxidativa son indicadores de EO celular y todos ellos pueden ser cuantificados ${ }^{8}$.

En el presente estudio la concentración hepática de DC y MDA estuvo significativamente aumentada en los ratones inyectados con DL-etionina, en comparación con los controles (Figuras 3A y 3B). Análogamente, se reporta la elevación de los niveles de MDA hepático en ratones expuestos a $\mathrm{CCl}$, conocido inductor de hepatoesteatosis ${ }^{1}$. Además, se ha indicado en ratones con dieta deficiente en metionina y colina, la presencia de una moderada esteatosis microvesicular del hígado, con elevación de la concentración hepática de triglicéridos y de MDA ${ }^{18}$.

Se debe añadir que en ratones carentes del gen que codifica a la metionina-adenosiltransferasa, se ha observado una reducción hepática del contenido de Sadenosilmetionina, lo que induce esteatosis y un estado de EO del hígado que se caracteriza por alta concentración de MDA, acompañada con disminución de las reservas antioxidantes, entre ellas el glutatión reducido ${ }^{9}$.

Ante el ataque oxidativo, el hepatocito se torna disfuncional con eventual muerte celular por necrosis y apoptosis, lo que hace inminente un fallo hepático. Se ha indicado paralelismo entre el daño hepático observado en ratones inyectados i.p. con $\mathrm{CCl}_{4}$, con la elevación de los niveles séricos de enzimas hepáticas tales como las aminotransferasas ALT y AST ${ }^{1}$, hallazgo coincidente con los resultados obtenidos en el presente estudio. Así se puede observar en la Figura 4, cómo en los ratones inyectados con DL-etionina las actividades plasmáticas de ALT y AST se encuentran extraordinariamente elevadas al compararlas con sus respectivos controles.

La utilización de productos naturales con propiedades antioxidantes en ratones expuestos al $\mathrm{CCl}_{4}$, ha logrado disminuir en forma significativa la actividad plasmática de ALT y AST, aunque sin recuperar el valor presentado por los ratones no expuestos al hepatotóxico ${ }^{6}$. 
El mal funcionamiento hepático aquí registrado se explica por el grave daño de los hepatocitos inducido por la DL-etionina, evidenciado en la Figura 1D. La instauración del estado de estrés oxidativo (Figuras 3A y 3B) abriría las puertas al proceso inflamatorio o esteatohepatitis que, al progresar, podría dañar las células y conducir a la fibrosis con formación de tejido cicatrizal cuya función no es la hepática. En un reciente estudio en ratas, se reveló que la ingestión de ácidos grasos lipoperoxidados desencadena un estímulo pro-inflamatorio en el hígado con aumento de la LPO y de las enzimas sintasas del óxido nítrico-2 (NOS-2) y ciclooxigenasa-2 (COX-2), como también de la interleukina $1 \beta$ (IL-1 $\beta$ ) y del factor de necrosis tumoral alfa $(\mathrm{TNF} \alpha)^{3}$.

Como conclusión, el hígado graso inducido por DL-etionina se acompaña con un estado de estrés oxidativo que compromete la funcionalidad hepática.

Agradecimientos. Los autores agradecen al Consejo de Desarrollo Científico, Humanístico y Tecnológico de la Universidad Centroccidental "Lisandro Alvarado" (UCLA, Venezuela) por el financiamiento otorgado a este estudio. Asimismo, a las Dras. Yaritza Salas y Victoria Colmenarez, del Laboratorio de Anatomía Patológica del Hospital Veterinario "Dr. Humberto Ramírez Daza" de la Facultad de Ciencias Veterinarias-UCLA, por la realización del diagnóstico histopatológico de las muestras hepáticas.

\section{REFERENCIAS}

1. Balahoroğlu R, Dülger H, Özbek H, Bayram I, Şekeroğlu MR. 2008. Protective effects of antioxidants on the experimental liver and kidney toxicity in mice. $E u$ J Gen Med 5: 157-164.

2. Bobe G, Young JW, Beitz DC. 2004. Invited review: pathology, etiology, prevention, and treatment of fatty liver in dairy cows. J Dairy Sci 87: 3105-3124.

3. Böhm T, Berger H, Nejabat M, Riegler T, Kellner F, Kuttke M, Sagmeister S, Bazanella M, Stolze K, Daryabeigi A, Bintner N, Murkovic M, Wagner KH, Schulte R, Rohr N, Huber W, Grasl B. 2013. Food-derived peroxidized fatty acids may trigger hepatic inflammation: a novel hypothesis to explain steatohepatitis. J Hepatol 59: 563-570.

4. Bradford MA. 1976. A rapid and sensitive method for the quantities of microgram of protein utilizing the principle of protein-dye binding. Analyt Biochem 72: 248-254.

5. Buqué X, Aspichueta P, Ochoa B. 2008. Fundamento molecular de la esteatosis hepática asociada a la obesidad (Molecular basis of obesity-related hepatic steatosis). Rev Esp Enferm Dig 100: 565-578.
6. Essawy AE, Abdel-Moneim AM, Latifa I. Khayyat LI, Elzergy AA. 2012. Nigella sativa seeds protect against hepatotoxicity and dyslipidemia induced by carbon tetrachloride in mice. J Appl Pharmac Sc 2: 21-25.

7. Fong DG, Nehra V, Lindor KD, Buchman AL. 2000. Metabolic and nutritional considerations in nonalcoholic fatty liver. Hepatology 32: 3-10.

8. Halliwell B, Gutterridge J. 1999. Free radicals in biology and medicine, Clarendon Press, Oxford, p. 149, 202230.

9. Martínez-Chantar ML, Corrales FJ, Martínez LA, García ER, Huang ZZ, Chen L, Kanel G, Avila MA, Mato JM, Lu SC. 2002. Spontaneous oxidative stress and liver tumors in mice lacking methionine adenosyltransferase-1-A. Faseb J 16: 1292-1294.

10. Ohkawa H, Ohishi N, Yagi K. 1979. Assay for lipid peroxides in animal tissues by thiobarbituric acid reaction. Analyt Biochem 95: 351-358.

11. Singal AK, Jampana SC, Weinman SA. 2011. Antioxidants as therapeutic agents for liver disease. Liver Internat 31: 1432-1448.

12. Shull C. 1966. On the mechanism of induction of hepatic adenosine triphosphate deficiency by ethionine. $J$ Biol Chem 241: 5060-5070.

13. Takahashi Y, Soejima Y, Fukusato T. 2012. Animal models of nonalcoholic fatty liver disease/nonalcoholic steatohepatitis. World J Gastroenterol 18: 2300-2308.

14. Wallin B, Rosengren B, Shertzer H, Camejo G. 1993. Lipoprotein oxidation and measurement of thiobarbituric acid reacting substances formation in a single microtiter plate: Its use for evaluation of antioxidants. Analyt Biochem 208: 10-15.

15. Weber C, Hametner C, Tuchscherer A, Losand B, Kanitz E, Otten W, Singh SP, Bruckmaier RM, Becker F, Kanitz W, Hammon HM. 2013. Variation in fat mobilization during early lactation differently affects feed intake, body condition, and lipid and glucose metabolism in highyielding dairy cows. J Dairy Sci 96: 165-180.

16. Wei Y, Rector S, Thyfault JP, Ibdah JA. 2008. Nonalcoholic fatty liver disease and mitochondrial dysfunction. World J Gastroenterol 14: 193-199.

17. Yuzefovych LV, Musiyenko SI, Wilson GL, Rachek LI. 2013. Mitochondrial DNA damage and dysfunction, and oxidative stress are associated with endoplasmic reticulum stress, protein degradation and apoptosis in high fat diet-induced insulin resistance mice. Plos One 8(1): e54059.

18. Zhang YK, Yeager RL, Tanaka Y, Klaassen CD. 2010. Enhanced expression of Nrf2 in mice attenuates the fatty liver produced by a methionine- and choline-deficient diet. Toxicol Appl Pharmacol 245: 326-334. 